hep-ph/0305071

DESY 03-054

\title{
Hadronic Cross-sections in two photon Processes at a Future Linear Collider ${ }^{1}$
}

\author{
R. M. Godbole ${ }^{a},{ }^{2}$ \\ Theory Division, DESY, Notkestrasse 85, D-22603 Hamburg, Germany \\ A. De Roeck ${ }^{b}$ \\ CERN, CH-1211, Geneva-23, Switzerland \\ A. $\mathrm{Grau}^{c}$ \\ CAFPE and DFTC, Universidad de Granada, Spain \\ G. Pancheri ${ }^{d}$ \\ Laboratori Nazionali di Frascati dell'INFN, Via E. Fermi 40, I 00044, Frascati, Italy.
}

\begin{abstract}
In this note we address the issue of measurability of the hadronic crosssections at a future photon collider as well as for the two-photon processes at a future high energy linear $e^{+} e^{-}$collider. We extend, to higher energy, our previous estimates of the accuracy with which the $\gamma \gamma$ cross-section needs to be measured, in order to distinguish between different theoretical models of energy dependence of the total cross-sections. We show that the necessary precision to discriminate among these models is indeed possible at future linear colliders in the Photon Collider option. Further we note that even in the $e^{+} e^{-}$option a measurement of the hadron production cross-section via $\gamma \gamma$ processes, with an accuracy necessary to allow discrimination between different theoretical models, should be possible. We also comment briefly on the implications of these predictions for hadronic backgrounds at the future TeV energy $e^{+} e^{-}$collider CLIC.
\end{abstract}

\footnotetext{
${ }^{1}$ E-mails: (a)rohini.godbole@desy.de, (b) deroeck@mail.cern.ch, (c) igrau@ugr.es (d) Giulia.Pancheri@lnf.infn.it

${ }^{2}$ Permanent Address: Centre for Theoretical Studies, Indian Institute of Science, Bangalore 560 012, India.
} 


\section{Introduction}

The rising total cross-section in proton-proton collisions was a very early indication of QCD processes at work, reflecting the fact that the increasing energy allows a deeper probe of the structure of the colliding particles, leading to liberation of more constituents which results in a higher scattering probability[1]. The proton-proton and proton anti-proton cross-sections are now known experimentally to a very good precision over a large energy range. We still do not have a full theoretical understanding of these cross-sections starting from first principles, but there are various models of hadronic interactions whose parameters can be completely fixed by the data and which then allow for good predictions of the total cross-section in the high energy region, certainly up to LHC energies. Thus, although not everything is calculable from first principles in QCD, the total hadronic production at future hadronic accelerators can be predicted. Deviations from these predictions, beyond the theoretical errors, could indicate the onset of new physics, just like the rise of total cross-section, first observed at the ISR[1], was indeed the signal of hitherto undetected partonic interactions.

The situation is quite different for the photon induced processes in that the data cover a smaller energy range and also have larger errors. This renders the issue of measurement of the total $\gamma \gamma$ cross-section at energies in the region 300-500 GeV, very important both from the theoretical, as well as an experimental point of view. Indeed, the question of hadron production in $\gamma \gamma$ collisions is interesting for achieving a good theoretical understanding of the rise of the hadronic cross-sections with energy, in the framework of QCD or otherwise, as well as from a much more pragmatic viewpoint of being able to estimate the hadronic backgrounds [2] at the next linear colliders, particularly in the photon collider option as well in the $e^{+} e^{-}$option like CLIC [3] in the higher energy range. HERA and LEP have opened the way to an entire new field in QCD, the study of the hadronic interactions of the photon in terms of its quark and gluon content [4]. The rise in the total hadronic cross section begins to take place at centre of mass energies below $100 \mathrm{GeV}$ but to determine the steepness of the rise one needs points in the range 300$500 \mathrm{GeV}$ and at even higher energies. These do not exist. Different models have been suggested in the context of rise of the hadronic cross-sections in $p p$ and $\bar{p} p$ processes. All of these 'explain' the rise for the $p p$ and $\bar{p} p$ case equally well but differ substantially in their predictions for $\gamma \gamma$ collisions even 
at the modest values of the $\gamma \gamma$ energies that are currently available. However, within various experimental uncertainities they are all compatible with the current data. Thus to gain a good theoretical understanding of the total cross-section for $\gamma \gamma$ processes, as in the case of hadronic collisions, one needs much higher energies and better statistics than the one currently available.

In the next section we discuss the available data on $\gamma \gamma$ total hadronic cross sections at high energies. In section 3 we introduce photon colliders and present recent model predictions for the cross-sections. Section 4 details the simulation study made to assess the possible precision of the experimental measurement of the hadronic cross-sections at the photon colliders. In the next sections we discuss the required precision in the measurement of total hadronic cross-sections so as to distinguish among different models at the photon colliders and $e^{+} e^{-}$colliders respectively and then we end with conclusions.

\section{Status of the currently available data and models}

The currently available experimental information on total hadronic crosssections for photon induced processes comes from the $e^{+} e^{-}$colliders PEP [5], PETRA[6] and LEP[7, 8] as well as HERA[9]. LEP and HERA provide the higher energy data. At LEP phase space limits the centre of mass system (CMS) energy, $\sqrt{s_{\gamma \gamma}}$, of the $\gamma \gamma$ interactions to about $100 \mathrm{GeV}$, whereas at HERA $\sqrt{s_{\gamma p}}$ is higher. However, at HERA the presence of the proton partly obscures the issue. Fig. 1 shows a collection of data for the total hadronic cross-section $\sigma(\gamma \gamma \rightarrow$ hadrons $)$ from the various $e^{+} e^{-}$experiments in comparison with the predictions from a number of theoretical models summarised in Ref. [10]. The predictions have been plotted from "proton-like" models, labelled SaS[11], Aspen[12], BSW[13], as well as from QCD and Regge inspired models, like the curve labelled GLMN[14] and the band labelled BKKS[15]. The band labelled EMM covers predictions of two different formulations, inelastic and total. For the EMM, we have used two sets of representative parameters[10], both of which are obtained from the $\gamma p$ cross-section following the procedure outlined in [16]. All models predict a rise of the crosssection with the collision energy $\sqrt{s_{\gamma \gamma}}$, but with very different slopes. Also 


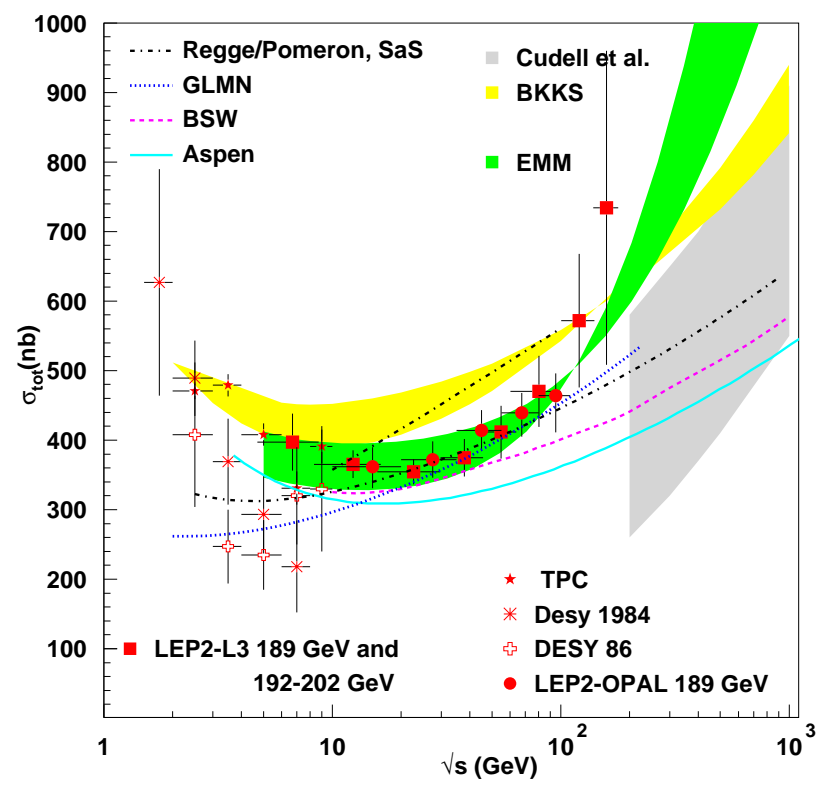

Figure 1: The predictions from factorization (proton like) models [11, 12, 13] Regge-Pomeron exchange[14] and a QCD structure function model [15] together with those from the EMM[10] are compared with the present data.

shown, by the region shaded in intermediate grey labelled Cudell et al, is a recent attempt of using only the low energy data to predict $\sigma_{\gamma p}$ and $\sigma_{\gamma \gamma}$ at TeV energies [17]. Within errors, their predictions are seen to cover the range spanned by the predictions of almost all the models discussed here. Further note that, until five years ago, the available data for $\gamma \gamma$ processes stopped short of $\sqrt{s}=20 \mathrm{GeV}$ and did not show any rise. The data in this energy range have very large errors, show a large spread and the compatibility of the different experiments is marginal. A re-measurement of this region, as planned at VLEP in Novisibirsk, may be very useful even for understanding the high energy region as will be shown below.

The L3 [7] and OPAL [8] data have drastically changed the situation. Presently, the $\gamma \gamma$ cross-section data indicate a very clear rise, which may be even stronger than in hadron-hadron collisions. This can be shown through 
Regge inspired fits of the type

$$
\sigma_{\gamma \gamma}^{\operatorname{had}}(s \gamma \gamma)=A s_{\gamma \gamma}^{\epsilon}+B s_{\gamma \gamma}^{-\eta}
$$

where $\epsilon$ and $\eta$ are expected to be process independent, i.e. the same in $p p$, $p \bar{p}, \gamma p$ and $\gamma \gamma$ collisions.

First we note that the recent data from LEP shown in Fig. 1 have been corrected with models for acceptance and particularly for the invisible elastic cross sections and the low acceptance diffractive processes. To this end, Monte Carlo simulation programs like PYTHIA[18] and PHOJET[19] have been used, which have different elastic and diffractive component predictions. The correction factors are different for these two and are large. Both L3 and OPAL follow now the same strategy to present their data, by taking the average of the results obtained with both models. The data of the experiments agree in the region of overlap.

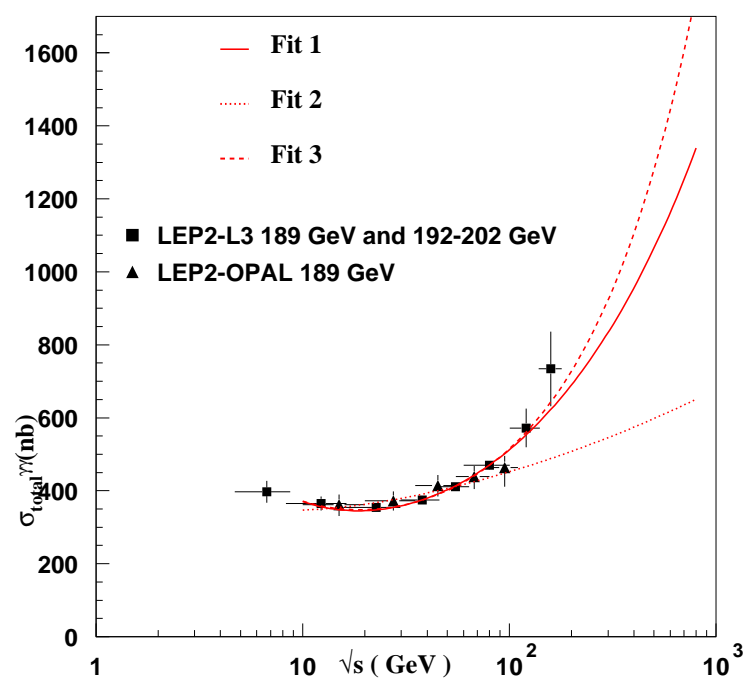

Figure 2: Data from OPAL and L3, shown with combined statistical and systematic error apart from the model dependence error, together with results from fits. In Fit 1 all parameters of Eq. 1 are free. In Fit 2, $\epsilon$ is fixed at the $p p / \bar{p} p$ value of 0.093 , other parameters are free and in Fit 3 a second pomeron term $C s^{\epsilon_{1}}$, with $\epsilon_{1}=0.418$ is added. 
Table 1: Results of fits to the OPAL and L3 total $\gamma \gamma$ cross sections, of the form $B s^{-\eta}+A s^{\epsilon}+C s^{\epsilon_{1}}$.

\begin{tabular}{|c||c|c|c|c|c||}
\hline Data & $A(\mathrm{nb})$ & $B(\mathrm{nb})$ & $C(\mathrm{nb})$ & $\epsilon, \epsilon_{1}$ & $\chi^{2}$ \\
\hline \hline L3 & $47 \pm 14$ & $1154 \pm 158$ & - & $\epsilon=0.250 \pm 0.033$ & 2.4 \\
L3 & $187 \pm 4$ & $312 \pm 95$ & - & $\epsilon=0.093$, fixed & 25 \\
L3 & $98 \pm 18$ & $958 \pm 162$ & $5.3 \pm 1.1$ & $\epsilon=0.093$, fixed & \\
& & & & $\epsilon_{1}=0.418$, fixed & 1.3 \\
L3+OPAL & $51 \pm 14$ & $1132 \pm 158$ & - & $\epsilon=0.240 \pm 0.032$ & 4.0 \\
L3+OPAL & $187 \pm 4$ & $310 \pm 91$ & - & $\epsilon=0.093$ fixed & 26 \\
L3+OPAL & $103 \pm 18$ & $934 \pm 156$ & $5.0 \pm 1.0$ & $\epsilon=0.093$, fixed & \\
& & & & $\epsilon_{1}=0.418$, fixed & 2.8 \\
\hline
\end{tabular}

Table 1 shows results of fits to the measurements in the form of Eq. 1, using the full errors (except for the model dependence), not taking into account correlations. Similar values for the parameters are obtained using only the statistical errors. Since the power of the Regge term and its size cannot be both determined from the LEP data alone, we take the value measured in $p p$ and $\gamma p$ interactions as given in the PDG[20], namely $\eta=0.358$. We then consider three cases:

- Fit1: All parameters $A, B$ and $\epsilon$ are left free

- Fit2: $\epsilon$ is fixed to 0.093, as measured in $p p$ and $\bar{p} p$ collisions, the other parameters are left free

- Fit3: $\epsilon$ is fixed to 0.093 , but a second pomeron term of the form $C s_{\gamma}^{\epsilon_{1}} \gamma$ as proposed in [21] was added with $\epsilon_{1}=0.418$ and the normalization $(C)$ fitted.

The fits are made for the L3 data alone - which have the largest range- and the L3+OPAL data. In the latter case the results remain dominated by the L3 data, but the relatively low increase in $\chi^{2}$ value shows that these data sets are now well compatible with each other. The results of the fit are shown in Fig. 2. Clearly the L3 data reject Fit2. A universal pomeron slope of about 0.08-0.093 is not compatible with the L3 data. Fit1 shows that the 
pomeron term is of order $0.2-0.3$. This remains true if the fits are made to the data corrected with either PHOJET or PYTHIA separately as the L3 collaboration reports in their paper [7]. Using two pomerons terms with a fixed power following [21], as in in Fit3, also accounts for the rise. Based on these data alone, and without taking into account correlations between the data points, the second pomeron component in the data is visible with about $5 \sigma$ significance. These results do not change significantly if the datapoint at the largest $\sqrt{s_{\gamma \gamma}}$ value is excluded from the fit. The results do show a dependence on the model used for unfolding the cross-sections. We find for the L3 data, that correcting the data using Pythia or Phojet at a time, instead of taking an average of both these models, yields values of $\epsilon$ of $0.29 \pm 0.03$ and $0.20 \pm 0.03$ respectively for Fit1. A similar model dependence in the fits was observed in [22]. Note however, that both values of $\epsilon$ are still significantly larger than the soft pomeron value of 0.093 . Thus the total $\gamma \gamma$ cross section appears to rise faster than in hadron-hadron collisions. It is however imperative that other LEP experiments make similar analyses in the high $\sqrt{s_{\gamma \gamma}}$ region to confirm this important result.

The results depend on the value of $\eta$ used in the fits. E.g. changing $\eta$ in the range of $0.3-0.45$ yields a slope $\epsilon$ of $0.280-0.215$ in Fit1 and the cross section for the second hard pomeron from $5.9 \pm 1.2-4.6 \pm 1.0$ in Fit3. While the main message of the fits to the data remains the same for these values, the exact value of the pomeron slope or cross section depends on the Regge power. To pin down the Regge part, better measurements at low energy in dedicated experiments will be needed.

Thus a study of high energy behaviour of the two-photon total cross section holds potential of yielding very interesting information. Improved or additional data in the LEP range from other experiments can help but there are a number of theoretical and experimental issues, which only an $e^{+} e^{-}$ Linear Collider (LC) can clarify, by reaching higher energies.

We now turn to discuss the models proposed so far for $\sigma_{\gamma \gamma}$. A review of the theoretical ideas and issues on the total cross section behaviour in $\gamma \gamma$ collisions was presented in [10]. As mentioned already, all models predict a rise of the cross-section with the collision energy $\sqrt{s_{\gamma \gamma}}$, but with very different slopes. The dramatic differences in the predictions for high energies show our present lack of understanding. In pure proton-like models (for example the solid curve [12]), the rise follows closely that of the proton-proton crosssection, while in QCD based models (upper [15] and lower [10] bands), the 
rise is obtained using the eikonalized pQCD jet cross-section. The upper and lower here refer to the position of the bands at low energy. In order to give a quantitative estimate of the energy dependence in the different models, one can fit the model predictions with eq.1 and calculate, for each model, the effective values of $\eta$ and $\epsilon$. The results for three of the models are given in Table 2. As expected, the slope for the Aspen model is close to that of

Table 2: Values of the parameters $A, B, \eta, \epsilon$ of Eq. 1 obtained by numerical fits to the various model predictions. The upper and lower refer to the position of the edge at high energies.

\begin{tabular}{|c||c|c|c|c||}
\hline Model & $A(\mathrm{nb})$ & $\epsilon$ & $B(\mathrm{nb})$ & $\eta$ \\
\hline \hline BKKS (upper edge) & 166.5 & 0.13 & 538.2 & 0.38 \\
BKKS (lower edge) & 180.6 & 0.11 & 356.5 & 0.18 \\
Aspen & 145.7 & 0.094 & 517.5 & 0.39 \\
\hline EMM (lower edge) & 14.01 & 0.34 & 475.4 & 0.14 \\
EMM (upper edge) & 19.9 & 0.29 & 475.3 & 0.084 \\
\hline
\end{tabular}

the proton cross-sections, while the QCD based models, EMM or BKKS, are described by $\epsilon$ values in the range $0.11-0.34$. Note, however, that the earlier discussion of the fits shows that the lower values of $\epsilon$ are in disagreement with the present data.

The discussion above just shows that data seem to indicate larger values for $\epsilon$ in eq. (1) for $\gamma \gamma$ processes, i.e. a faster rise with energy than for the $p p / p \bar{p}$ processes. In addition, we also see that different models differ greatly in their predictions. All this makes it clear that a good measurement of the total hadronic cross-section for photon induced processes is quite important. In the next sections we will examine the experimental issues involved in measuring two-photon interactions at a future high energy $\mathrm{e}^{+} \mathrm{e}^{-}$and $\gamma \gamma$ collider, extending previous estimates up to energy of $1 \mathrm{TeV}$ in the $\gamma \gamma$ centre of mass frame. 


\section{Linear colliders and Two Photon Physics.}

Two photon processes at future high energy LC can be measured either as in a storage ring, via photon emission from the lepton beams, according to a Weizsäcker Williams(WW)[23] energy distribution, or using the LC in a photon collider mode [24]. In the latter case the high energy electron beam is converted into a high energy photon beam, by backscattering of photons off an intense laser beam, just before the interaction point. The maximum energy of the generated photons is given by $E_{\gamma}^{\max }=x E_{e} /(1+x)$, with $E_{e}$ the electron beam energy and $x=4 E_{e} E_{L} \cos ^{2}(\theta / 2) / m_{e}^{2} c^{4}$ with $E_{L}$ and $\theta$ the laser photon energy and angle between the electron and laser beam. The distance of the conversion to the interaction point is in the range of several $\mathrm{mm}$ to a few $\mathrm{cm}$. A typical value for $x$ is 4.8 , which leads to photon spectra which peak around $0.8 E_{e}$. Hence, a typical distribution of $\gamma \gamma$ luminosity as a function of the invariant mass peaks at the maximum

reachable invariant mass of around $0.8 \sqrt{s_{e^{+} e^{-}}}$with a width of $\approx 0.10-0.15$ for $\gamma \gamma$ collisions. The 'luminosity' is usually defined to be the luminosity corresponding to the region $0.8 \sqrt{s_{\gamma \gamma, \max }}<\sqrt{s_{\gamma \gamma}}<\sqrt{s_{\gamma \gamma, \max }}$ and is typically $10 \%$ of the geometrical $e^{+} e^{-}$luminosity. However, since the electron beams are converted before collision, smaller $\beta^{*}$ functions at the interaction point can be allowed for. This can make up for part of the luminosity lost compared to an $e^{+} e^{-}$collider, by using smaller beam spots. For TESLA [25], for an LC with initial CMS energy of $500 \mathrm{GeV}$, one finds [26] $\mathcal{L}_{\gamma \gamma} \simeq 0.35 \mathcal{L}_{e^{+} e^{-}}$ This leads to event samples corresponding to a luminosity of the order 100 $\mathrm{fb}^{-1}$ per year, for the Photon Collider. Similar numbers are obtained for CLIC [27].

In this study the PHOJET [19] program was used which has an option for a simple backscattered laser (BL) photon spectrum. Both the $\mathrm{e}^{+} \mathrm{e}^{-}$and BL modes of the LC were investigated. Detector effects were studied with the program SIMDET [28].

\section{Simulation study}

First we consider the case for the $\mathrm{e}^{+} \mathrm{e}^{-}$collider mode. Present data for the two photon cross-section at LEP are hampered by experimental and detector limitations. For two photon events coming from interactions of quasi-real 
photons, the electrons disappear in the beam-pipe. Hence the only information available is the hadronic final state. The variable $\sqrt{s_{\gamma \gamma}}$ needs to be reconstructed from the visible hadronic final state in the detector. At the highest energies for a $500 \mathrm{GeV} e^{+} e^{-} \mathrm{LC}$ the hadronic final state extends in pseudorapidity $\eta=\ln \tan \theta / 2$ in the region $-8<\eta<8$, as shown in Fig. 3a, while a typical LC detector covers roughly the region $-3<\eta<3$. Hence the correlation of the measured $\sqrt{s_{\gamma \gamma}}$ compared to the true one will be even poorer at the LC than at LEP, as shown in Fig. 3b. However, some information can be obtained by measuring the total integrated cross-section above a value $\sqrt{s_{\gamma \gamma}}($ see Section 5$)$.

For a $\gamma \gamma$ collider the photon beam energy can be tuned with a spread of less than $10 \%$, such that measurements of $\sigma_{\gamma \gamma}^{\text {tot }}$ can be made at a number of "fixed" energy values in e.g. the range $100<\sqrt{s_{\gamma \gamma}}<400 \mathrm{GeV}$ by changing the beam energy of the collider, as shown in Fig. 3c. For the spectra shown it was assumed in the simulation that the same value of $x$ is kept at each energy, which means that the wavelength of the laser needs to be changed at each beam energy. A more likely scenario is that the same laser is used at the lower energies, which will then have correspondingly different $x$ values, and thus somewhat different spectra.

In the simulation study events were selected with visible $\sqrt{s_{\gamma \gamma}}>10 \mathrm{GeV}$, and it was asumed that a minimum of three charged tracks would be detected in the experiment. The acceptance for non-diffractive events was found to be about $95 \%$. The simulation shows that the diffractive non-elastic events are accepted with $35 \%$ efficiency, while elastic events are essentially lost. Hence to measure the total cross-section with sufficient precision specially designed measurements for the diffractive components will be required. To that end also measurements at lower energy (e.g. LEP) will be useful, in order to extrapolate the elastic component. A technique to measure diffractive contributions separately, mirrored to the rapidity gap methods used at HERA, has been proposed in [29]. It is assumed here that such measurements will be made, and it will be possible to reduce the systematic error due to the diffractive component. The absolute precision with which these cross-sections can be measured ranges from $5 \%$ to $10 \%$ for collider like TESLA, in the CMS energy range up to $700 \mathrm{GeV}$. The important contributions to the errors come from the control of the diffractive component of the cross-section, Monte Carlo models used to correct for the event selection cuts, the knowledge of absolute luminosity and shape of the luminosity spectrum. For a photon 

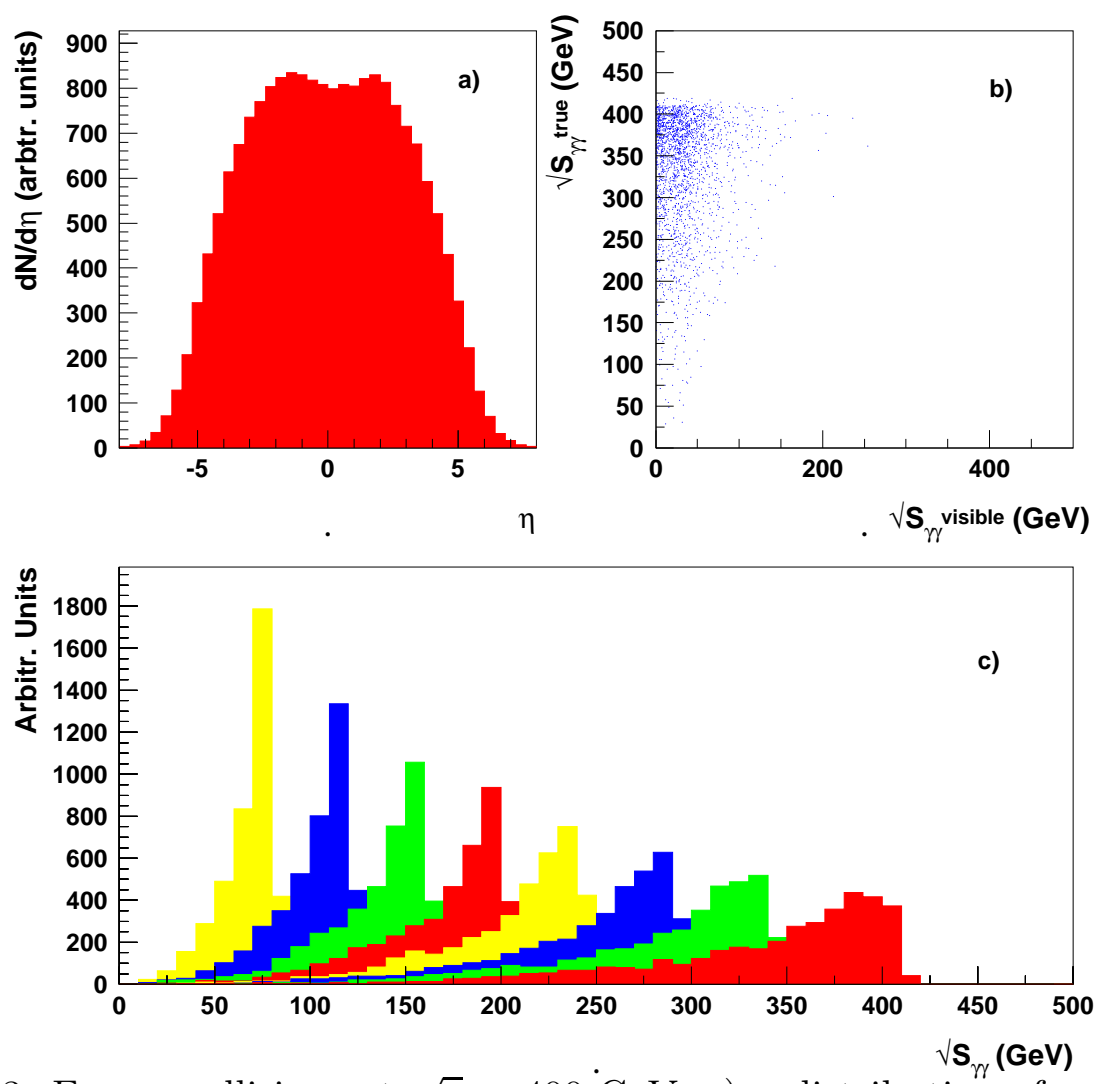

Figure 3: For $\gamma \gamma$ collisions at $\sqrt{s}=400 \mathrm{GeV}$ : a) $\eta$ distribution of produced particles; b) correlation between the true and visible $\sqrt{s}$; c) luminosity spectra for eight measurements of $\sigma_{\text {tot }}$ at different energies.

collider based on CLIC [27], which could reach $\sqrt{s_{\gamma \gamma}}$ values up to $2 \mathrm{TeV}$ and beyond, the corresponding error range is from about 7 to $15 \%$. The errors given in Table. 3 are estimated using SIMDET [28] and extrapolations from HERA and LEP studies.

Fig. 4 shows present photon-photon cross-section data in comparison with predictions at higher photon energies from some of the recent phenomenological models [10] illustrated in Fig. 1. Because of uncertainties in the overall normalization, some of the curves reproduced in this figure have been scaled (up or down) so as to overlap with the low energy data point from LEP experiments. This emphasizes solely the energy dependence and reduces the uncertainties due to the low energy normalization. Pseudo data points from 
Table 3: List of systematic errors for the total cross-section measurement. The third column comments on the source/reason

\begin{tabular}{|c||c|c||}
\hline Type & Value & Comment \\
\hline \hline Selection cuts & $3 \%$ & $\sim$ HERA $/$ frag. parameters \\
Diffraction & $3-8 \%$ & $\rho \rho$ events \\
Detector smearing & $3 \%$ & $\sim$ HERA \\
\hline Lumi $/ E_{\gamma}$ spectrum & $3-4 \%$ & short runs $(\sim$ one day $)$ \\
Bin correction & $2 \%$ & $\sqrt{s_{\gamma \gamma}}$ spread \\
\hline
\end{tabular}

one of (thus scaled) models [15] are added with a systematic error of 7\%$11 \%$. The statistical errors are small since even only for a day of running at a given energy, $O\left(10^{7}\right)$ events are produced. Statistical samples of order $10^{5}$ events will be sufficient for this measurement.

\section{Precision needed to distinguish between models}

In this section we update previous estimates $[10,30]$ for the precision needed to distinguish between different models for the total $\gamma \gamma$ cross-section. In

Table 4: Expected $\gamma \gamma$ cross-sections and precision required for in their measurement to distinguish between the different 'proton' like models

\begin{tabular}{|c||c|c|c|c||}
\hline$\sqrt{s_{\gamma \gamma}}(\mathrm{GeV})$ & Aspen & BSW & DL & $1 \sigma$ \\
\hline \hline 20 & $309 \mathrm{nb}$ & $330 \mathrm{nb}$ & $354 \mathrm{nb}$ & $7 \%$ \\
50 & $330 \mathrm{nb}$ & $368 \mathrm{nb}$ & $402 \mathrm{nb}$ & $10 \%$ \\
100 & $362 \mathrm{nb}$ & $401 \mathrm{nb}$ & $450 \mathrm{nb}$ & $10 \%$ \\
200 & $404 \mathrm{nb}$ & $441 \mathrm{nb}$ & $507 \mathrm{nb}$ & $9 \%$ \\
500 & $474 \mathrm{nb}$ & $515 \mathrm{nb}$ & $598 \mathrm{nb}$ & $8 \%$ \\
700 & $503 \mathrm{nb}$ & $543 \mathrm{nb}$ & $636 \mathrm{nb}$ & $8 \%$ \\
1000 & $538 \mathrm{nb}$ & $578 \mathrm{nb}$ & $679 \mathrm{nb}$ & $7 \%$ \\
\hline
\end{tabular}




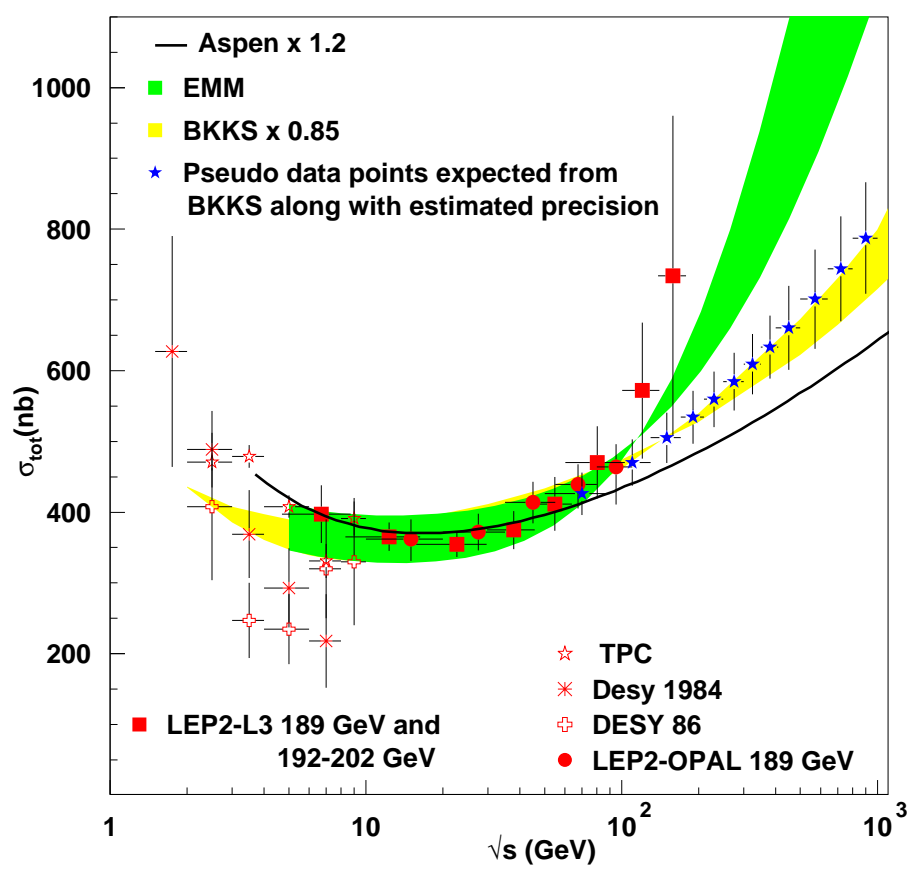

Figure 4: The total $\gamma \gamma$ cross-section as function of the collision energy, compared with model calculations: BKKS band (upper and lower limit correspond to different photon densities [15]); a proton-like model (solid line [12]); EMM band (Eikonal Minijet Model for total and inelastic cross-section, with different photon densities and different minimum jet transverse momentum [10]). The proton-like and BKS models have been normalized to the data, in order to show the energy dependence of the cross-section.

Table 4 we show total $\gamma \gamma$ cross-sections for three models of the "photon-islike-the-proton' type. The last column shows the $1 \sigma$ level precision needed to discriminate between Aspen[12] and BSW[13] models. The model labelled DL is obtained from Regge/Pomeron exchange and factorization at the residues [31], with parameters from ref.[20]. Table 5 gives the precision needed to distinguish between the two minijet formulations of Fig. 1 and the BKKS model[15]. The last column in Table 5 gives the percentage difference between the two models which bear closest results among these three.

A detailed comparison of the predictions reveals that in order to distinguish between all the models the cross-sections need to be determined to a precision of better than $10 \%$ [10] at a future $0.5-1 \mathrm{TeV} \mathrm{e}^{+} \mathrm{e}^{-}$collider. This 
Table 5: Expected $\gamma \gamma$ cross-sections and precision required in their measurement in order to distinguish between different formulations of the EMM and BKKS [15], whose common characteristic is that the high energy rise is computed with a QCD input.

\begin{tabular}{|c||c|c|c|c||}
\hline$\sqrt{s_{\gamma \gamma}}(\mathrm{GeV})$ & $\begin{array}{c}\text { EMM, Inel,GRS } \\
\left(p_{\text {tmin }}=1.5 \mathrm{GeV}\right)\end{array}$ & $\begin{array}{c}\text { EMM, Tot,GRV } \\
\left(p_{\text {tmin }}=2 \mathrm{GeV}\right)\end{array}$ & $\begin{array}{c}\text { BKKS } \\
\text { GRV }\end{array}$ & $1 \sigma$ \\
\hline \hline 20 & $399 \mathrm{nb}$ & $331 \mathrm{nb}$ & $408 \mathrm{nb}$ & $2 \%$ \\
50 & $429 \mathrm{nb}$ & $374 \mathrm{nb}$ & $471 \mathrm{nb}$ & $9 \%$ \\
100 & $486 \mathrm{nb}$ & $472 \mathrm{nb}$ & $543 \mathrm{nb}$ & $3 \%$ \\
200 & $596 \mathrm{nb}$ & $676 \mathrm{nb}$ & $635 \mathrm{nb}$ & $6 \%$ \\
500 & $850 \mathrm{nb}$ & $1165 \mathrm{nb}$ & $792 \mathrm{nb}$ & $7 \%$ \\
700 & $978 \mathrm{nb}$ & $1407 \mathrm{nb}$ & $860 \mathrm{nb}$ & $13 \%$ \\
1000 & $1133 \mathrm{nb}$ & $1694 \mathrm{nb}$ & $940 \mathrm{nb}$ & $19 \%$ \\
\hline
\end{tabular}

seems feasible from the simulation studies discussed above. It should be pointed out that some of the differences in the model predictions even at higher energies are due to the low energy normalization. If all the models were to be scaled down to the same value, for instance at $50 \mathrm{GeV}$ c.m. energy, then differences among the different proton like models would very much decrease. Not so for the QCD based models, which continue to show very different high energy behaviour.

On the other hand, while the absolute cross-sections are measured with limited precision, the change of the cross-section with energy can be determined much more accurately. Fitting the data of the collider to the Regge inspired form $s^{\epsilon}$ in the high energy region, one can determine $\epsilon$ with a precision of $\Delta \epsilon=0.02$. The models show a variation between $\epsilon=0.08$ and $\epsilon=0.26$ and hence can be distinguished.

\section{$6 \quad \sigma_{t o t}$ in the $e^{+} e^{-}$mode}

It will be difficult to measure $\sigma_{\text {tot }}$ with sufficient precision in the normal $e^{+} e^{-}$ mode of the linear collider, however some information can be gained from 
measuring the cross-section $\sigma\left(e^{+} e^{-} \rightarrow e^{+} e^{-}\right.$hadrons $)$. This is calculated by convoluting the $\gamma \gamma$ total cross-section with the spectrum of these photons. This spectrum is given by the Weizsäcker Williams(WW) or effective photon approximation[23] which has been used quite successfully to translate photoproduction cross-sections into those for electron initiated processes. There have been many discussions of the improvements on the original WW approximation [32]. This has also been extended to include the effects of a reduction in the parton content of the photon due to virtuality of the photon [33], while dealing with the resolved photon processes. The cross-section, including the effects due to (anti)tagging of the electron is given by

$$
\sigma_{e^{+} e^{-}}^{\text {had }}=\int_{z \min }^{1} d z_{1} \int_{z \min / z_{1}}^{1} d z_{2} f_{\gamma / e}\left(z_{1}\right) f_{\gamma / e}\left(z_{2}\right) \sigma(\gamma \gamma \rightarrow \text { hadrons }) .
$$

Here $z_{\text {min }}=s_{\text {min }} / s$ where $\sqrt{s}$ is the c.m. energy of the $e^{+} e^{-}$collider. The WW spectrum used is given by

$$
f_{\gamma / e}(z)=\frac{\alpha_{\mathrm{em}}}{2 \pi z}\left[\left(1+(1-z)^{2}\right) \ln \frac{P_{\max }^{2}}{P_{\min }^{2}}-2(1-z)\right],
$$

where

$$
P_{\max }^{2}=s / 2 *\left(1-\cos \theta_{t a g}\right)(1-z), P_{\min }^{2}=m_{e}^{2} \frac{z^{2}}{(1-z)} .
$$

Here, using $\theta_{\text {tag }}$ the maximal scattering angle for the outgoing electron, we have taken anti-tagging into account and have included the suppression of the photonic parton densities due to its virtuality following ref. [34].

To select $e^{+} e^{-} \rightarrow e^{+} e^{-}$hadrons events, a minimum value of $s_{\gamma \gamma}$ is required, selecting a region such that the value of $s_{\gamma \gamma}$ can be corrected for smearing and losses with sufficient precision. Also a maximum value is imposed, because the events resemble annihilation events for too large a value of $s_{\gamma \gamma}$ and cannot be easily separated. Additionally an anti-tagging condition for the scattered electrons is imposed. SIMDET simulation studies lead to choose the region $50 \mathrm{GeV}^{2}<s_{\gamma \gamma}<0.64 s_{e e}$, and the anti-tagging cuts are $\theta_{\mathrm{tag}}=0.025, E_{\text {min }}^{e}=$ $0.2 E_{\text {beam }}$. With these cuts the total cross-section can be determined with a precision of $5-10 \%$.

In Fig. 5, we show the cross-section as a function of $\sqrt{s}$ of the $e^{+} e^{-}$ machine. The top curve corresponds to the prediction for $\sigma_{\gamma \gamma}$ of the BKKS model [15] and the lower curve corresponds to the prediction of the Aspen 


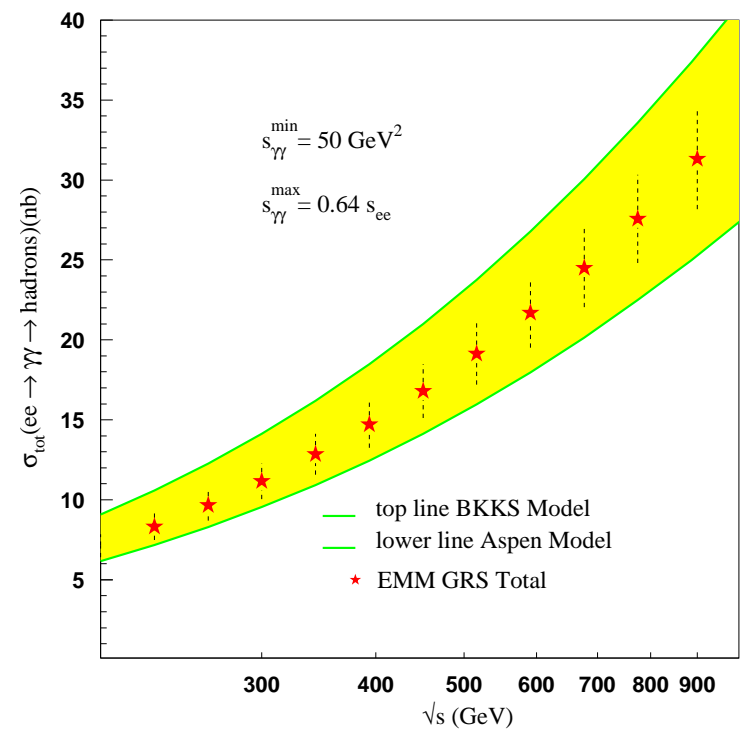

Figure 5: Cross-sections for hadron production due to $\gamma \gamma$ interactions in $e^{+} e^{-}$reactions.

model [12]. Note that the difference of about factor 2 (say) at $\sqrt{s}=700$ $\mathrm{GeV}$, is reduced to about $30-40 \%$ after convolution with the bremsstrahlung spectrum. The 'data points' are given with a pessimistic error of $10 \%$. Hence even in the worst of the cases (no photon collider, large uncertainties in the $e^{+} e^{-} \sigma_{\gamma \gamma}^{t o t}$ cross-section measurement) a linear collider with $\sqrt{s_{e^{+} e^{-}}}=500$ $\mathrm{GeV}$, can definitively contribute to a further understanding of the energy dependence of the total $\gamma \gamma$ cross-section.

Eq. 2 can also be used to calculate the number of hadronic events per bunch crossing, which is expected to be significant at the high energy $e^{+} e^{-}$ collider like CLIC. However, in this case it is necessary to add the effect of the beamstrahlung photons as well. We do this for CLIC, by taking the spectra of beamstrahulng photons as provided in Ref. [35]. For the design parameters considered, the two-photon luminosities per bunch crossing, corresponding to both or one photon being a bremsstrahlung one are $\mathcal{L}_{e e}^{\gamma \gamma}=6.4852 \times 10^{34} \mathrm{~m}^{-2}$ and $\mathcal{L}_{\text {eg }}^{\gamma \gamma}=5.3589 \times 10^{34} \mathrm{~m}^{-2}$ respectively, where as the one coming from beamstrahlung photons alone is $\mathcal{L}_{g g}^{\gamma \gamma}=4.9534 \times 10^{34} \mathrm{~m}^{-2}$. The expected number of hadronic events expected per bunch crossing, with these effective 
Table 6: Number of events per bunch crossing expected at CLIC.

\begin{tabular}{|c||c|c|c|c||}
\hline$s_{\min } \mathrm{GeV}^{2}$ & Aspen & EMM(BN) & BKKS & EMM \\
\hline \hline 5 & 4. & 5.5 & 5.7 & 6.3 \\
25 & 3.4 & 4.7 & 5.0 & 5.5 \\
50 & 3.2 & 4.5 & 4.7 & 5.3 \\
\hline
\end{tabular}

two photon luminosities per bunch crossing, are shown in Table 6. It shows the number of events expected for three different values of the lower limit on $s_{\gamma \gamma}$ instead of the fixed value of $50 \mathrm{GeV}^{2}$ considered above.

The number obtained by us for the Aspen model is consistent with that in Ref.[36] with SAS parametrisation of the $\sigma_{\text {tot }}(\gamma \gamma \rightarrow$ hadrons). Thus we see that depending on which theoretical model gives the right high energy description, we expect between 4-7 hadronic events per bunch crossing at CLIC. The beamstrahlung photons completely dominate the $\gamma \gamma$ luminosity. Inclusion of the beamstrahlung contribution increases the expected number of events by about a factor 10 than expected just for the bremsstrahlung photons. However, about half of these events come from the contribution to the $\gamma \gamma$ luminosity coming from the cross-term between the bremsstrahlung and the beamstrahlung photons.

\section{Conclusions}

Future linear $e^{+} e^{-}$colliders will be instrumental in the study of high energy photon collisions. We have shown that it will be possible at these colliders, to extract new important information on the energy dependence of the total hadronic cross-section in two photon collisions, $\sigma_{\gamma \gamma}^{\text {had }}$. The best option for this purpose would be the photon collider mode, allowing for precise measurements at several $\sqrt{s_{\gamma \gamma}}$ energies. Accuracies of the order of $5-10 \%$ can be achieved, in a region where model predictions vary by a factor 2 or more. Even in the $e^{+} e^{-}$mode, there is sufficient sensitivity left in the total inclusive reaction $e^{+} e^{-} \rightarrow e^{+} e^{-}$hadrons (which does not require reconstruction of $\sqrt{s_{\gamma \gamma}}$ ), to distinguish between model classes though perhaps not between models in a given class. Further, it should be added that, for centre of mass 
energies of the $e^{+} e^{-}$collider up to a $800-1000 \mathrm{GeV}$, the size of this inclusive cross-section is determined more by the normalisation of $\sigma(\gamma \gamma \rightarrow$ hadrons $)$ for $\sqrt{s_{\gamma \gamma}}$ in $100-200 \mathrm{GeV}$ region than by the steepness of its energy dependence. Thus clarification of the measurements by the LEP groups in this energy range can play a crucial role in the determination of the hadronic backgrounds per bunch crossing expected in the $e^{+} e^{-}$mode.

\section{Acknowledgments}

This work was supported in part by the EU through HPRN-CT2002-00311. RMG wishes to thank the CERN theory division for hospitality where part of this work was done and the Department of Science and Technology, India for partial support under project no. SP/S2/K-01/2000-II.

\section{References}

[1] D.Cline, F.Halzen and J. Luthe, Phys. Rev. Lett. 31 (1973) 491.

[2] M. Drees and R.M. Godbole, Phys. Rev. Lett. 67 (1991) 1189; P. Chen, T. Barklow and M.E. Peskin, Phys. Rev. D49 (1994) 3209, R.M. Godbole, hep-ph/9807379, Proceedings of the Workshop on Quantum Aspects of Beam Physics, Jan. 51998 - Jan. 9 1998, Monterey, U.S.A., 404-416, Ed. P. Chen, World Scientific, 1999.

[3] CLIC, A linear collider based on CLIC technology, the CLIC study team, G. Guignard (Editor), CERN-2000-08.

[4] For a review, see for example, M. Drees and R.M. Godbole, Journal of Phys. G21 (1995) 1559.

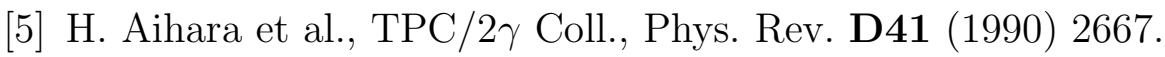

[6] S.E. Baru et al., MD-1 Coll., Zeit. Phys. C53 (1992) 219.

[7] L3 Collaboration, M. Acciarri, et al,, CERN-EP/2001-012, Phys. Lett. B519 (2001) 33, hep-ex/0102025.

[8] OPAL Collaboration. G. Abbiendi et al., Eur. Phys. J. C14 (2000) 199. 
[9] H1 Collaboration, S. Aid, et al., Zeit. Phys. C69, 27 (1995), hepex/9405006;

ZEUS collaboration, S. Chekanov et al., Nucl. Phys. B627 (2002) 3, hep-ex/0202034.

[10] R. M. Godbole, G. Pancheri, Eur. Phys. J. C19 (2001) 129, hep$\mathrm{ph} / 0010104$.

[11] G. Schuler and T. Sjöstrand, Zeit. Phys. C68 (1995) 607; Phys. Lett. B376 (1996) 193; Zeit Phys. C73 (1997) 677.

[12] M.M. Block, E.M. Gregores, F. Halzen and G. Pancheri, Phys.Rev. D58 (1998) 17503; M. Block, E.M. Gregores, F. Halzen and G. Pancheri, Phys.Rev. D60 (1999) 54024.

[13] C. Bourelly, J. Soffer and T.T. Wu, Mod.Phys.Lett. A15 (2000) 9.

[14] E. Gotsman, E. Levin, U. Maor, E. Naftali, Eur.Phys.J. C14 (2000) 511, hep-ph/0001080.

[15] B. Badelek, M. Krawczyk, J. Kwiecinski and A.M. Stasto, Phys.Rev. D62 (2000) 074021; e-Print Archive: hep-ph/0001161.

[16] A. Corsetti, R.M. Godbole and G. Pancheri, Phys.Lett. B435 (1998) 441.

[17] J.R. Cudell et al., hep-ph/0212101.

[18] T. Sjöstrand, Comp. Phys. Comm., 82 (1994) 74;

T. Sjöstrand, Lund University report, LU-TP-95-20 (1995).

[19] R. Engel and J. Ranft, Phys. Rev. D54 (1996) 4244;

R. Engel, Zeit. Phys. C66 (1995) 203.

[20] D.E. Groom et al, PDG 2000, Eur. Phys. Jour. C15, (2000), 1.

[21] A. Donnachie and P. Landshoff, Phys. Lett. B437 (1998) 408.

[22] M. Block and K. Kang, hep-ph/0302146.

[23] C.F. v. Weizsäcker, Z. Phys. 88,(1934) 612; E.J. Williams, Phys. Rev. 45 (1934) 729. 
[24] I.F. Ginzburg et al., Nucl. Inst. Meth. A205 (1983) 47, A219 (1984) 5;

V.I. Telnov, Nucl. Inst. Meth. A94 (1990) 72, hep-ex/9908005.

R. Brinkmann et al., Nucl. Instrum. Meth. A406 (1998) 13.

[25] TESLA, R. Brinkman et al., DESY-01-011 and ECFA-2001-209.

[26] V. Telnov, hep-ex/0101002.

[27] H. Burkhardt, V. Telnov, CERN-SL-2002-013-AP, CLIC-NOTE-508, May 2002

[28] M. Pohl and J. Schreiber, DESY99-030 (1999).

[29] A. De Roeck, R. Engel and A. Rostovtsev, hep-ph/9710366.

[30] R. Godbole, A. Grau and G. Pancheri, QCD and Multiparticle Production, page 424. Proceedings of the XXIX International Symposium on Multiparticle Dynamics, Brown U., Aug. 2000. Eds. I. Sarcevic and C-I Tang. World Scientific 2000. e-print Archive: hep-ph/9912395.

[31] A. Donnachie and P.V. Landshoff, Phys. Lett. B296 (1992) 227.

[32] See for example, S. Frixione, M.L. Mangano, P. Nason and G. Ridolfi, Phys. Lett. B319 (1993) 339.

[33] M. Drees and R.M. Godbole, Phys. Rev. D50 (1994) 3124.

[34] M. Drees and R.M. Godbole, Zeit. Phys. C59 (1993) 591.

[35] D Schulte, private communication and http://clicphysics.web.cern.ch/CLICphysics/

[36] D. Schulte, DESY-TESLA-97-08, 1997 